\title{
Análise energética do novo sistema de produção de maracujá amarelo na região de Marília-SP
}

\author{
Energy analysis of new production system of yellow passion in region of Marilia-SP, Brazil
}

\author{
Fernanda de Paiva Badiz Furlaneto ${ }^{I^{*}}$ Maura Seiko Tsutsui Esperancini ${ }^{I I}$ Adriana Novais Martins ${ }^{\mathrm{I}}$ \\ Fumiko Okamoto ${ }^{\mathrm{I}}$ Anelisa de Aquino Vidal $^{\mathrm{I}}$ Osmar de Carvalho Bueno ${ }^{\mathrm{II}}$
}

RESUMO

Objetivou-se avaliar indicadores energéticos de um novo sistema de produção do maracujá amarelo na região de Marília-SP. Foram analisadas as "entradas" de energia de origem biológica, fóssil e industrial e a "saída” energética na forma de frutos produzidos por unidade de área, bem como cinco indicadores energéticos. A entrada energética totalizou 155.810,13 MJ/ha. Os adubos químicos e defensivos responderam por $71 \%$ e $24 \%$ da energia indireta industrial, respectivamente. A atividade gerou, por ciclo/ha, 587.700,00MJ, sendo a produtividade cultural $0,19 \mathrm{MJ} \mathrm{kg}^{-1}$. A eficiência cultural foi de 3,77. A energia cultural líquida totalizou 431.889,87 MJ/ha. A eficiência energética (4.17) e o balanço energético (550.312,91MJ ha-1) foram positivos. Concluiu-se que o consumo de energia direta de origem fóssil, mesmo que significativo, ainda permite a produção do fruto de maneira ambientalmente sustentável. Os adubos químicos $e$ defensivos foram os itens mais representativos da energia indireta industrial, em decorrência da intensificação da adubação como forma de prevenção de doenças.

Palavras-chave: Passiflora edulis $f$. flavicarpa, eficiência energética, balanço de energia.

\section{ABSTRACT}

The study aimed to evaluate energy indicators of a new production system of yellow passion fruit in Marília-SP. Analyzed the "inputs" of the biological energy, fossil and industrial and "output" energy in the form of fruits produced per unit area as well as five energy indicators. The input energy amounted $155,810.13 \mathrm{MJ} \mathrm{ha}^{-1}$. Chemical fertilizers and pesticides accounted for $71 \%$ and $24 \%$ of indirect energy industry, respectively. The activity generated per cycle / ha, 587.700.00MJ, and cultural productivity $0.19 \mathrm{MJ} \mathrm{kg}^{-1}$. The efficiency culture was 3.77. The cultural energy net totaled 431,889.87MJ ha-1. The energy efficiency (4.17) and energy balance $\left(550,312.91 \mathrm{MJ} \mathrm{ha}^{-1}\right)$ was favorable. It was concluded that the direct energy consumption from fossil fuels, even though significant, yet permits the production of the fruit of an environmentally sustainable manner. Chemical fertilizers and pesticides were the most representative of indirect energy industry due to the intensification of fertilization as a means of disease prevention.

Key words: Passiflora edulis f. flavicarpa, energy efficiency, energy balance.

\section{INTRODUÇÃO}

A fruticultura é, atualmente, um dos segmentos mais importantes da agricultura brasileira, respondendo por $25 \%$ do valor da produção agrícola nacional. O Brasil é o maior produtor mundial de maracujá, com aproximadamente $60 \%$ da produção total (IBGE, 2012). No país, a produção do fruto destaca-se na região Nordeste, Sudeste e Norte. $\mathrm{Na}$ região Sudeste, o maracujazeiro é uma das oito espécies frutíferas mais cultivadas no sistema extensivo (MELETTI, 2011). A cultura do maracujá vem se destacando como uma alternativa agrícola para a pequena propriedade por utilizar, predominantemente, mão de obra familiar, com expressivo valor social, gerando um capital de giro durante vários meses do ano (ARAÚJO NETO et al., 2009).

GLIESSMAN (2001) enfatiza que a sustentabilidade dos agroecossistemas decorre da convergência entre ações que envolvem aspectos econômicos, ambientais e sociais. Assim, é importante, também, mensurar o aporte de energia

\footnotetext{
IAgência Paulista de Tecnologia dos Agronegócios (APTA), Unidade de Pesquisa e Desenvolvimento de Marília, Bairro Cascata, 17515-400, Marília, SP, Brasil. E-mail: fernandafurlaneto@apta.sp.gov.br.*Autor para correspondência.

IFaculdade de Ciências Agronômicas (FCA), Universidade Estadual Paulista "Júlio de Mesquista Filho" (UNESP), Botucatu, SP, Brasil. Recebido 28.01.13 Aprovado 29.08.13 Devolvido pelo autor 23.11.13 CR-2013-0110.R1
} 
usado direta e indiretamente na cultura para avaliar a viabilidade da atividade ao longo do tempo.

O objetivo deste estudo foi avaliar os indicadores energéticos da produção do maracujá amarelo (Passiflora edulis) na região de Marília-SP, em um novo sistema de plantio, com uso de mudas altas.

\section{MATERIAL E MÉTODOS}

Foram analisados municípios situados na área de abrangência do Escritório de Desenvolvimento Rural de Marília-SP/Coordenadoria de Assistência Técnica Integral (EDR/CATI), sendo eles: Álvaro de Carvalho, Alvinlândia, Fernão, Gália, Garça, Lupércio, Marília, Ocauçu, Oriente, Oscar Bressane, Pompéia, Quintana e Vera Cruz. O levantamento de campo foi realizado no período de janeiro de 2011 a julho de 2012. A escolha dos municípios para a coleta dos dados energéticos seguiu a metodologia descrita por CASTRO \& PEREIRA (2000).

A região de Marília situa-se na região Centro Oeste Paulista e caracteriza-se por apresentar $69 \%$ das unidades de produção agrícola (UPA) com áreas de até 50 hectares. O clima, segundo a Carta Climática do Estado de São Paulo, baseada no sistema de Köppen, é do tipo Cwa, com verão quente e inverno seco, a temperatura média do mês mais quente superior a $23^{\circ} \mathrm{C}$ e a do mês mais frio entre $-3^{\circ} \mathrm{C}$ e $18^{\circ} \mathrm{C}$. A precipitação pluviométrica média anual é $1.129 \mathrm{~mm}$, com deficiência hídrica anual da ordem de 50 a $80 \mathrm{~mm}$. O solo predominante na região é classificado como ArgissoloVermelhoAmarelo eutrófico abrúptico, textura arenosa/média, característico da forma de relevo suave ondulado a ondulado na região, com declives da ordem de 3 a $15 \mathrm{~cm} \mathrm{~m}^{-1}$ (BERTOLANI et al., 2000).

O sistema tradicional de plantio do maracujá ocorre no mês de fevereiro, com uso de muda convencional (tubete), ciclo de produção de 18 meses, espaçamento de $3,5 \mathrm{~m}$ entre linhas x 3,0m entre plantas, densidade de $952 \mathrm{pl}$. ha ${ }^{-1}$, produtividade de $20 \mathrm{t} \mathrm{ha}^{-1}$, período de colheita de novembro a julho e destino da produção, sendo $60 \%$ para mesa e $40 \%$ para indústria.

Nos meses de março e abril, ocorre revoada de pulgões, período de maior incidência de focos de virose, principalmente a do endurecimento dos frutos. Dessa forma, optou-se por avaliar um novo sistema de plantio, visando a reduzir o ataque de virose por meio da alteração da época de formação do pomar, bem como otimizar a produtividade e qualidade dos frutos.

Os indicadores fitotécnicos avaliados nesse novo sistema produtivo foram: mês de plantio: agosto; tipo de muda: muda alta (saco plástico); altura média da muda: 1,5m; ciclo de produção: 12 meses; espaçamento: $3,5 \mathrm{~m}$ entre linhas x 2,5m entre plantas; densidade: 1.142pl. ha-1; produtividade: $30 \mathrm{tha}^{-1}$; período de colheita: janeiro a julho; comercialização: $70 \%$ para mesa e $30 \%$ para indústria.

As variáveis analisadas foram baseadas em dados técnicos de outros pólos produtores do fruto (região da Alta Sorocabana - Presidente Prudente e municípios limítrofes) e ajustadas para o local em estudo.

A matriz de coeficientes técnicos de produção do maracujá na região de Marília foi elaborada com base em informações coletadas por meio da aplicação de questionários junto a fruticultores.

$\mathrm{Na}$ região do estudo, encontram-se 153 propriedades que cultivam maracujá, totalizando, aproximadamente, 275 hectares. Foram selecionadas 12 (doze) propriedades para realização da análise energética do cultivo de maracujazeiros. A escolha das propriedades considerou o sistema de implantação e condução da lavoura (tecnologia representativa regionalmente) e destino da produção (comercial).

A análise energética do maracujazeiro amarelo seguiu a metodologia apresentada por RISOUD (1999), que avalia a dependência de energia não renovável de um sistema. Foram consideradas as "entradas" de energia referentes à mão de obra, calcário, adubo químico e orgânico, muda, combustível, óleo lubrificante, graxa, operações de máquinas e materiais de consumo demandados durante o ciclo produtivo e a "saída" energética na forma de fruto produzido por unidade de área. A unidade adotada para o estudo energético foi o megajoule (MJ). O índice de conversão do joule (J) em caloria (cal) correspondeu a 0,24, ou seja, $1 \mathrm{MJ}$ equivale a $238,84 \mathrm{kcal}$.

A determinação da energia investida pelos trabalhadores nas diversas operações que caracterizam o itinerário técnico da cultura do maracujá considerou a média do valor do GER (Gasto Energético no Repouso) apresentado por BUENO (2002) na atividade de calagem $\left(0,7 \mathrm{MJ} \mathrm{h}^{-1}\right)$, em função de o dispêndio energético do trabalho executado nesta atividade aproximar-se das realizadas pelos funcionários no cultivo de maracujá.

$\mathrm{O}$ índice energético do calcário correspondeu a $0,17 \mathrm{MJ} \mathrm{kg}^{-1}$. Para a determinação do conteúdo energético dos fertilizantes, multiplicouse a quantidade efetiva do elemento ativo pelo valor energético correspondente, sendo $\mathrm{N}, \mathrm{P}_{2} \mathrm{O}_{5}$ e $\mathrm{K}_{2} \mathrm{O}$ igual a $63,79,13,97,9,76 \mathrm{MJ} \mathrm{kg}^{-1}$, respectivamente 
(ZANINI et al., 2003). O valor utilizado para o adubo orgânico $\left(14,70 \mathrm{MJ} \mathrm{kg}^{-1}\right)$ foi baseado em SANTOS \& LUCAS JUNIOR (2004).

Os valores usados para os herbicidas $\left(228 \mathrm{MJ} \mathrm{kg}^{-1}\right)$, inseticidas e acaricidas $(309,94 \mathrm{MJ}$ $\left.\mathrm{kg}^{-1}\right)$, fungicidas e antibiótico $\left(272,14 \mathrm{MJ} \mathrm{kg}^{-1}\right)$ e formicidas (194MJ $\mathrm{kg}^{-1}$ ) foram obtidos na descrição de CAMPOS (2001) e OLIVEIRA JUNIOR (2005). Os índices energéticos dos mourões de eucalipto e bambu corresponderam a $0,16 \mathrm{MJ} \mathrm{kg}^{-1}$ e $0,11 \mathrm{MJ}$ $\mathrm{kg}^{-1}$, respectivamente (BAUER, 2001). Os itens arame $\left(0,63 \mathrm{MJ} \mathrm{kg}^{-1}\right)$, esticador $\left(0,40 \mathrm{MJ} \mathrm{kg} \mathrm{kg}^{-1}\right)$ e tela de proteção $\left(0,30 \mathrm{MJ} \mathrm{kg}^{-1}\right)$ foram adaptados de DOERING (1980).

O consumo do combustível, óleo lubrificante e graxa (energia direta de origem fóssil) seguiu os valores determinados por PACHECO (2000) e KAMPHORST (2003), sendo o consumo médio correspondente a 7 litros de óleo diesel por hora de serviço; 7 litros de óleo de motor a cada 200 horas de serviço; $0,5 \mathrm{~kg}$ de graxa a cada 10 de horas de serviço (trator); e $0,3 \mathrm{~kg}$ de graxa a cada 10 horas de serviço (implemento). $\mathrm{O}$ índice energético utilizado refere-se a $40,8 \mathrm{MJ} \mathrm{L}^{-1}$ para o óleo diesel, $37,7 \mathrm{MJ} \mathrm{L}^{-1}$ para o óleo lubrificante e $43,3 \mathrm{MJ} \mathrm{kg}^{-1}$ para a graxa (BRASIL, 2012).

A demanda específica de energia (DEE) das máquinas e implementos foi baseada nos dados apresentados por ASSENHEIMER et al. (2009), adaptados de DOERING (1980), sendo eles: trator $2,51 \mathrm{MJ} \mathrm{h}^{-1}$, roçadeira $0,19 \mathrm{MJ} \mathrm{h}^{-1}$, pulverizador de barras $0,46 \mathrm{MJ} \mathrm{h}^{-1}$, carreta $0,75 \mathrm{MJ}$ h, sulcador $0,22 \mathrm{MJ}$ $\mathrm{h}^{-1}$, atomizador $0,34 \mathrm{MJ} \mathrm{h}^{-1}$, tanque $0,68 \mathrm{MJ} \mathrm{h}^{-1}$.

$\mathrm{O}$ valor energético da muda de maracujá e a "saída" de energia do pomar, que representa a energia do fruto produzido por unidade de área, foram calculados por meio de análises laboratoriais efetuadas na UNESP, Campus de Botucatu, no Laboratório de Física Aplicada do Departamento de Física e Biofísica do Instituto de Biociência.

Para tanto, foram coletadas quatro amostras de muda alta e cinco frutos em cada propriedade analisada. Os pesos médios das mudas e dos frutos, em massa seca, foram, respectivamente: $19,75 \mathrm{~g}$ e 204,11g.

Após a coleta de campo, realizou-se o preparo das amostras, que foram encaminhadas para avaliação na bomba calorimétrica (SILVA, 1981). As etapas para o preparo compreenderam duas fases: 1) moagem do material in natura e 2) desidratação de $100 \mathrm{~g}$ de cada amostra em estufa com circulação e renovação de ar contínua a uma temperatura de $65^{\circ} \mathrm{C}$, durante 72 horas.
Os indicadores energéticos avaliados corresponderam ao balanço energético, eficiência energética, produtividade cultural, eficiência cultural e energia cultural líquida, representados da seguinte maneira:

a) Produtividade cultural $(\operatorname{PrC})=$ quantidade de produto / "entradas" culturais

em que: quantidade de produto $=$ produtividade por unidade de área $\left(\mathrm{kg} \mathrm{ha}^{-1}\right)$;

"entradas" culturais = energia da mão de obra, insumos e operações de máquinas por

unidade de área $\left(\mathrm{MJ} \mathrm{ha}^{-1}\right)$.

b) Eficiência cultural (EfC) = "saídas" úteis / "entradas" culturais

em que: "saídas" úteis = energia produzida pelo fruto por unidade de área $\left(\mathrm{MJ} \mathrm{ha}^{-1}\right)$.

c) Energia cultural líquida $($ EfCL) = "saídas" úteis "entradas" culturais.

d) Balanço energético $(\mathrm{BE})=\Sigma$ energias totais $-\Sigma$ "entradas" de energia não renováveis em que: energias totais = "entradas culturais".

e) Eficiência energética $(\mathrm{EfE})=\Sigma$ das energias totais / $\Sigma$ das "entradas" de energias não renováveis.

\section{RESULTADOS E DISCUSSÃO}

A entrada energética totalizou 155.810,13MJ ha-1. Os itens mais representativos corresponderam à energia indireta industrial (38\%) e biológica (38\%). Os adubos químicos e defensivos responderam por $71 \%$ e $24 \%$ da energia indireta industrial, respectivamente. Esses valores indicaram que a sustentabilidade da atividade está diretamente ligada com o manejo nutricional e fitossanitário da cultura (Tabela 1).

Identificou-se o valor energético médio de $0,38 \mathrm{MJ}_{\text {muda }^{-1}}$ alta e 3,99MJ fruto ${ }^{-1}$, ou seja, 19,59MJ $\mathrm{kg}^{-1}$ fruto. Portanto, o poder calorífico médio das mudas, por hectare, correspondeu a $1.260 \mathrm{MJ}$. A "saída" energética foi equivalente a $587.700 \mathrm{MJ} \mathrm{ha}^{-1}$.

Os valores apresentados neste trabalho são superiores aos encontrados por SALLA et al. (2009) no cultivo da cana-de-açúcar (14.370,9MJ ha-1). No entanto, esses autores, também, identificaram que os gastos energéticos mais significativos foram com insumos (50\%), que, juntamente com a condução da lavoura (14\%) e a colheita (13\%), somaram $77 \%$ do consumo energético total.

OLIVEIRA et al. (2007) identificaram que o custo energético total da produção agrícola do milho safrinha correspondeu a $12.982,71 \mathrm{MJ} \mathrm{ha}^{-1}$ na alta tecnologia e $8.414,69 \mathrm{MJ} \mathrm{ha}^{-1}$ na baixa tecnologia, sendo que o item mais oneroso foi o de "insumos" 
Tabela 1 - "Entradas" energéticas no cultivo de maracujazeiro amarelo, plantio com muda alta, por ciclo/hectare, em MJ, de acordo com o tipo, fonte e forma, região de Marília-SP, 2012

\begin{tabular}{|c|c|}
\hline Tipo, fonte e forma & "Entradas" culturais \\
\hline \multicolumn{2}{|l|}{ Energia Direta } \\
\hline \multicolumn{2}{|l|}{ Biológica } \\
\hline Mão de obra & 660,45 \\
\hline Mudas & 481,77 \\
\hline Eucalipto & 24,00 \\
\hline Bambu & 24,50 \\
\hline Subtotal & $1.190,72$ \\
\hline \multicolumn{2}{|l|}{ Fóssil } \\
\hline - Óleo diesel & $35.414,40$ \\
\hline - Lubrificante & 163,62 \\
\hline - Graxa & $1.809,07$ \\
\hline Subtotal & $37.387,09$ \\
\hline \multicolumn{2}{|l|}{ Energia Indireta } \\
\hline \multicolumn{2}{|l|}{ Biológica } \\
\hline \multicolumn{2}{|l|}{ Adubo orgânico } \\
\hline Esterco curral & $58.800,00$ \\
\hline Subtotal & $58.800,00$ \\
\hline \multicolumn{2}{|l|}{ Industrial } \\
\hline Calcário & 340,00 \\
\hline Total & 340,00 \\
\hline \multicolumn{2}{|l|}{ Adubo químico } \\
\hline Fósforo (Yorin) & 577,50 \\
\hline Nitrato de amônio & $32.640,00$ \\
\hline Cloreto de potássio & $8.292,80$ \\
\hline Ácido bórico & 4,32 \\
\hline Sulfato de zinco & 16,20 \\
\hline Subtotal & $41.530,82$ \\
\hline \multicolumn{2}{|l|}{ Defensivos } \\
\hline Fungicida 1 & $15.239,84$ \\
\hline Fungicida 2 & $10.613,46$ \\
\hline Inseticida 1 & 805,84 \\
\hline Acaricida 1 & 805,84 \\
\hline Antibiótico 1 & 408,21 \\
\hline Glifosato (herbicida) & $1.140,00$ \\
\hline Mirex (formicida) & 388,00 \\
\hline Subtotal & $14.161,36$ \\
\hline \multicolumn{2}{|l|}{ Máquinas e implem. } \\
\hline Trator & 311,24 \\
\hline Roçadeira & 3,80 \\
\hline Pulverizador barras & 0,92 \\
\hline Carreta & 25,50 \\
\hline Sulcador & 0,88 \\
\hline Atomizador & 19,72 \\
\hline Tanque & 4,08 \\
\hline Subtotal & 366,14 \\
\hline \multicolumn{2}{|c|}{ Mat. consumo em geral } \\
\hline Arame & $1.890,00$ \\
\hline Esticador & 24,00 \\
\hline Tela de proteção & 120,00 \\
\hline Subtotal & $2.034,00$ \\
\hline Total & $155.810,13$ \\
\hline
\end{tabular}

(76\%). As operações que mais apresentaram custos energéticos na cultura do milho safrinha foram as que demandaram mais horas de serviço (capina, semeadura/adubação e pulverização) e utilizaram grandes quantidades de recursos não-renováveis (defensivos e fertilizantes). No sistema de média tecnologia, a adubação de cobertura não foi realizada e os fungicidas usados no tratamento de sementes, por serem menos energéticos, reduziram o consumo de energia durante o processo produtivo. Os valores energéticos dos nutrientes foram altos em decorrência da presença de nitrogênio na sua formulação. $O$ óleo diesel representou, respectivamente, $20 \%$ e $30 \%$ do custo energético no sistema de alta e média tecnologia, indicando a necessidade de utilização de fontes energéticas alternativas para a minimização dos gastos energéticos.

Na cultura da soja, ASSENHEIMER et al. (2009) verificaram que o consumo energético para a produção de um hectare foi de 12.254,44MJ (cultivo orgânico) e $16.723,43 \mathrm{MJ}$ (cultivo convencional). $\mathrm{O}$ maior dispêndio de energia foi decorrente do fertilizante (cultivo orgânico), com 5.408,63MJ ha-1, ou seja, $44 \%$ do total de energia consumida. No cultivo convencional, o herbicida respondeu por $8.837,73 \mathrm{MJ}$ $\mathrm{ha}^{-1}$, representando $53 \%$ da energia consumida. O componente biológico (mão de obra) participou com apenas $3 \%$ e $1 \%$ do total de energia inserida no cultivo orgânico e convencional, respectivamente.

Destaca-se que,nocultivodemaracujazeiros, o alto consumo energético advém do manejo intensivo do pomar, como, por exemplo, as pulverizações de defensivos semanais que sobrecarregam o sistema quantitativamente e qualitativamente, em relação à sustentabilidade ambiental. Não ocorre monitoramento de pragas e doenças. Há escassez de assistência técnica especializada na cultura. Pesquisas regionais avaliam novas recomendações técnicas (adensamento de plantio) em decorrência da redução do ciclo de produção.

Verificou-se, na cultura do maracujazeiro amarelo, plantio com muda alta, na região de MaríliaSP, uma produtividade cultural correspondente a $0,19 \mathrm{MJ} \mathrm{kg}^{-1}$. A eficiência cultural foi de 3,77. A energia cultural líquida totalizou 431.889,87MJ ha-1.

$\mathrm{O}$ balanço energético que mostra a diferença entre as energias totais e "entradas" de energias não renováveis foi positivo no sistema avaliado $\left(550.312,91 \mathrm{MJ} \mathrm{ha}^{-1}\right)$. No entanto, esse valor pode ser otimizado com a redução do uso de energia fóssil não renovável.

A eficiência energética mostrou-se favorável, apresentando um valor de 4,17 (Tabela 2). 
Esse valor indica que a relação entre a somatória das energias totais e a somatória das "entradas" de energia não renovável é positiva. Vê-se, portanto, que o consumo de energia direta de origem fóssil, mesmo que significativa no sistema avaliado, ainda permite a produção do fruto de maneira ambientalmente sustentável.

No entanto, deve-se buscar sempre utilizar fontes energéticas alternativas para a minimização dos gastos energéticos para que a atividade se mantenha sustentável em longo prazo (AGOSTINHO \& ORTEGA 2012). Uma das possibilidades referese à intensificação da adoção de adubação orgânica, uso de combustível de origem biológica, como por exemplo, o biodiesel e introdução de monitoramento e pragas e doenças visando reduzir o gasto com defensivos químicos (FRANZESE et al., 2009).

\section{CONCLUSÃO}

O cultivo de maracujazeiro amarelo na região de Marília-SP, no sistema de plantio com muda alta, pode ser considerado como uma fonte alternativa de diversificação da empresa rural, sob o ponto de vista energético.

Os adubos químicos e defensivos foram os itens mais representativos da energia indireta industrial, em decorrência da intensificação da adubação como forma de prevenção de doenças e uso preventivo e curativo de fungicidas, inseticidas, acaricidas. Esses valores mostraram que a sustentabilidade da atividade está diretamente ligada com o manejo nutricional e fitossanitário da cultura, problemas estes que devem ser minimizados para

Tabela 2 - Indicadores energéticos do sistema de produção do maracujazeiro amarelo, plantio com muda alta, por ciclo, região de Marília-SP, 2012.

\begin{tabular}{lcc}
\hline Indicador & Unidade & $\begin{array}{l}\text { Plantio } \\
\text { muda alta }\end{array}$ \\
\hline Entrada "útil" & $\mathrm{MJ} \mathrm{ha}^{-1}$ & $155.810,13$ \\
Saída "útil" & $\mathrm{MJ} \mathrm{ha}^{-1}$ & $587.700,00$ \\
Produtividade cultural (PrC) & $\mathrm{MJ} \mathrm{ha}^{-1}$ & 0,19 \\
Eficiência cultural (EfC) & & 3,77 \\
Energia cultural líquida (EfCL) & $\mathrm{MJ} \mathrm{ha}^{-1}$ & $431.889,87$ \\
Balanço energético (BE) & $\mathrm{MJ} \mathrm{ha}^{-1}$ & $550.312,91$ \\
Eficiência energética (EfE) & & 4,17 \\
\hline
\end{tabular}

Obs: PrC = quantidade de produto / "entradas" culturais; EfC = "saídas" úteis / "entradas" culturais; EfCL = "saídas" úteis "entradas" culturais; BE $\equiv$ energias totais - $\Sigma$ “entradas" de energia não renováveis; EfE $\Sigma$ das energias totais $/ \Sigma$ das “entradas" de energias não renováveis possibilitar o desenvolvimento da fruticultura, a médio e longo prazo.

Os indicadores energéticos, em especial, a eficiência e o balanço energético, apontaram que a atividade permite a produção do fruto de maneira ambientalmente sustentável.

\section{REFERÊNCIAS}

AGOSTINHO, F; ORTEGA, E. Integrated food, energy and environmental services production as na alternative for small rural properties in Brazil. Energy, Philadelphia, v.37, p.103-114, 2012. Disponível em: <http://www.sciencedirect.com/science/journal /03605442/37/1>. Acesso em: 12 ago. 2013.

ARAÚJO NETO, S.E. et al. Produtividade e vigor do maracujazeiroamarelo plantado em covas e plantio direto sob manejo orgânico. Ciência Rural, Santa Maria, v.39, p.678-683, 2009. Disponível em: <http://www.scielo.br/pdf/cr/v39n3/a106cr395.pdf>. Acesso em: 02 ago. 2013

ASSENHEIMER, A. et al. Análise energética de sistemas de produção de soja convencional e orgânica. Ambiência, Guarapuava, v.5, n.3, p.443-455, 2009. Disponível em: <http:// http://rioverde.ifgoiano.edu.br/periodicos/index.php/gst/article/ view/43/83>. Acesso em: 10 jan. 2013.

BAUER, S.R.T. Resíduos da exploração florestal de Eucalyptus grandis para geração de energia elétrica. 2001. 54f. Dissertação (Mestrado em Energia na Agricultura) - Curso de Agronomia, Faculdade de Ciência Agronômicas, Universidade Estadual Paulista, Botucatu, SP.

BERTOLANI, F.C. et al. Levantamento pedológico semidetalhado do Estado de São Paulo: Folha de Marília, Memorial Descritivo. Campinas: Instituto Agronômico, 2000. 142p.

BRASIL. Ministério de Minas e Energia. Balanço energético nacional. Brasília, DF, 2012. 168p.

BUENO, O.C. Análise energética e eficiência do milho em assentamento rural, Itaberá-SP. 2002. 146f. Tese (Doutorado em Energia na Agricultura) - Curso de Agronomia, Faculdade de Ciências Agronômicas, Universidade Estadual Paulista, Botucatu, SP.

CAMPOS, A.T. Balanço energético relativo à produção de “coast-cross" e alfafa em sistema de produção de leite. 2001. 267f. Tese (Doutorado em Energia na Agricultura) - Curso de Agronomia, Faculdade de Ciências Agronômicas, Universidade Estadual Paulista, Botucatu, SP.

CASTRO, A.M.G.; PEREIRA, J.P. Estudo de caso a cadeia produtiva da borracha no Brasil. In: HOEFLICH, V. et al. Curso de especialização em engenharia da produção: gestão rural e agroindustrial. Florianópolis: UFSC-LED/SENAR-PR, 2000. p.131-179.

DOERING, O.C. Accouting for energy in farm machinery and buildings. In: PIMENTEL,D. (Ed.). Handbook of energy utilization in agriculture. Boca Raton, Flórida: CRC, 1980. p.9-14.

FRANZESE, P.P. et al. Sustainable biomass production: a comparison between gross energy requirement and emergy synthesis methods. Ecological Indicators, Germany, v.9, n.5, p.959-970, 
2009. Disponível em: <http://www.sciencedirect.com/science/ article/pii/S1470160X0800160X>. Acesso em: 14 ago. 2013.

GLIESSMAN, S.R. The ecological foundation of agroecosystem sustainability. Boca Raton: CRC, 2001. 198p.

INSTITUTO BRASILEIRO DE GEOGRAFIA E ESTATÍSTICA (IBGE). Pesquisa de orçamentos familiares, dados demográficos e produção agrícola municipal. Rio de Janeiro, 2012. Disponível em: $<$ http://www.sidra.ibge.gov.br $>$. Acesso em: 05 fev. 2013.

KAMPHORST, J.S. Combustíveis: consumo. Revista Cultivar Máquinas, Pelotas, n.10, p.8-10, 2003.

MELETTI, L.M.M. Avanços na cultura do maracujá no Brasil. Revista Brasileira de Fruticultura, Jaboticabal, v.33, n. esp, p.83-91, 2011. Disponível em: <http://dx.doi.org/10.1590/S010029452011000500012>. Acesso em: 10 jun. 2013.

OLIVEIRA JUNIOR, E.D. Análise energética de dois sistemas de colheitas mecanizadas de eucalipto. 2005. 74f. Tese (Doutorado em Agronomia) - Escola Superior de Agricultura "Luiz de Queiroz”, Universidade de São Paulo, Piracicaba, SP.

OLIVEIRA, M.M. et al. Análise da eficiência energética e econômica dos sistemas de produção de milho safrinha no Médio Paranapanema, Estado de São Paulo. In: CONGRESSO BRASILEIRO DE ENGENHARIA AGRÍCOLA, 36., 2007. Bonito, MS. Anais... Bonito: SBEA, 2007. 1 CD.
PACHECO, E.P. Seleção e custo operacional de máquinas agrícolas. Rio Branco: Embrapa Acre, 2000. 21p.

RISOUD, B. Développement durable et analyse énergétique d'exploitations agricoles. Économie Rurale, Paris, n.252, p.16-26, 1999. Disponível em: <http://www.persee.fr/web/revues/home/ prescript/article/ecoru 0013-0559 1999 num 252 1 5096>. Acesso em: 14 jan. 2013.

SALLA, D.A. et al. Avaliação energética da produção de etanol utilizando como matéria-prima a cana-de-açúcar. Ciência Rural, Santa Maria, v.39, n.8, p.2516-2520, 2009. Disponível em: $<$ http:// www.scielo.br/pdf/cr/v39n8/a307cr1202.pdf $>$. Acesso em: 18 jan. 2013. doi: 10.1590/S0103-84782009005000170.

SANTOS, T.M.B.; LUCAS JUNIOR, J. Balanço energético em galpão de frangos de corte. Engenharia Agrícola, Jaboticabal, v.24, n.1, p.25-36, 2004. Disponível em: <http://www.scielo.br/ pdf/eagri/v24n1/v24n01a04.pdf $>$. Acesso em: 22 dez. 2012. doi: 10.1590/S0100-69162004000100004.

SILVA, D.J. Análise de alimentos: métodos químicos e biológicos. Viçosa: UFV, 1981. 166p

ZANINI, A. et al. Análise do consumo de energia na produção de silagem de milho em plantio direto. Revista Acta Scientiarum Animal Sciences, Maringá, v.25, n.2, p.249-253, 2003. Disponível em: <http://periodicos.uem.br/ojs/index.php/ActaSciAnimSci / article/view/1980>. Acesso em: 08 jan. 2013. doi: 10.4025/ actascianimsci.v25i2.1980 